\title{
Active Retrograde Extra Backup with a Mother-and-Child Catheter to Facilitate Retrograde Microcatheter Collateral Channel Tracking in Recanalization of Coronary Chronic Total Occlusion
}

\author{
Yong Wang $\mathbb{D}^{\mathbb{D}}$, Xiao-Jiao Zhang, Hong-Wei Zhao, Cheng-Fu Wang, De-Feng Luo, \\ Qing-Kun Meng, Yu Zhu, Jie Tao, Bao-Jun Chen, Yi Li, Ai-Jie Hou $\mathbb{D}^{\text {, }}$, and Bo Luan
}

Department of Cardiology, The People's Hospital of China Medical University, The People's Hospital of Liaoning Province, Shenyang, China

Correspondence should be addressed to Ai-Jie Hou; 1758624242@qq.com and Bo Luan; luanbo2016@163.com

Received 4 May 2020; Revised 24 July 2020; Accepted 16 August 2020; Published 31 August 2020

Academic Editor: William B. Hillegass

Copyright (C) 2020 Yong Wang et al. This is an open access article distributed under the Creative Commons Attribution License, which permits unrestricted use, distribution, and reproduction in any medium, provided the original work is properly cited.

\begin{abstract}
Objective. To explore the feasibility and safety of the active retrograde backup (ARB) for treatment of chronic total occlusion (CTO) during retrograde percutaneous coronary intervention (PCI). Background. Guiding support plays an important role in guidewire and microcatheter coronary channel (CC) tracking in retrograde PCI therapy for patients with CTO. However, the feasibility and safety of retrograde active use of a mother-and-child catheter are still unclear. Patients and Methods. A total of 271 consecutive patients with CTO who underwent retrograde PCI between January 2015 and January 2020 were retrospectively analyzed. Clinical data of two groups were compared to evaluate the feasibility and safety of ARB. Results. Of the 271 patients, $69.0 \%$ (187/271) underwent therapy through the septal branch, 31.0\% (84/271) through the epicardial collateral channel, and 47.6\% (129/271) through active retrograde extra backup with a mother-and-child catheter to facilitate retrograde microcatheter collateral CC tracking. The time of wire CC tracking was shorter in the ARB group than that in the non-ARB group (25.4 \pm 8.5 vs $26.4 \pm 9.7, p=0.348)$, but there was no significant difference. The duration of the retrograde microcatheter tracking $(10.2 \pm 3.8$ vs $15.5 \pm 6.8, p=0.012)$ and the retrograde approach $(62.8 \pm 20.3$ vs $70.4 \pm 24.3, p=0.026)$ in the ARB group was significantly shorter than that in the non-ARB group. The radiation dose (223.6 \pm 112.7 vs. $295.2 \pm 129.3, p=0.028)$, fluoroscopy time $(50.6 \pm 21.3$ vs $62.3 \pm 32.1, p=0.030)$, and contrast volume $(301.8 \pm 146.7$ vs $352.2 \pm 179.5, p=0.032)$ in the ARB group were significantly lower than that in the non-ARB group. There were no life-threatening procedural complications in either group. Complications unrelated to ARB included two cases of donor-vessel dissection, one case of CC perforation, and two cases of target-vessel perforation. There was no statistically significant difference in major adverse cardiac and cerebrovascular events between the groups during hospitalization $(p>0.05)$. Conclusion. ARB is feasible, safe, and conducive to guidewire and microcatheter CC tracking in the recanalization of coronary CTO. It improves procedural efficiency and is worthy of further promotion.
\end{abstract}

\section{Introduction}

Chronic total occlusion (CTO) lesions occur in $16-18 \%$ of patients with coronary artery disease undergoing coronary angiography $[1,2]$. Before the introduction of the retrograde approach, the success rate of revascularization of CTO lesions was approximately $70 \%$ [3]. However, with advances in devices and new techniques, the most important being the introduction of the retrograde approach, the probability of success is above $90 \%$ at present. Nevertheless, retrograde interventional therapy for CTO-percutaneous coronary intervention (PCI) still faces great challenges. The primary retrograde approach may be considered as an alternative in patients who failed in antegrade CTO-PCI or those who have good collateral circulation.

The core of the retrograde interventional therapy is microcatheter tracking after successful guidewire tracking. In some cases, even if the guidewire passes through, 
retrograde microcatheter coronary channel (CC) tracking remains a challenge. Clinically, we found that the failure of microcatheter tracking in some patients was related to the poor supporting ability of retrograde guiding, and in cases where the retrograde guidewire has passed through the collateral circulation, replacing guiding is not the first choice. Retrograde passive delivery of a mother-and-child catheter to enhance support is a good approach, but it can lead to longer procedural time and increased X-ray exposure. Therefore, completing microcatheter CC tracking quickly and efficiently is important. In this study, we evaluated the feasibility and safety of Guidezilla (a motherand-child catheter)-based active retrograde extra backup-termed active retrograde backup (ARB) - to facilitate retrograde microcatheter collateral channel tracking in the recanalization of CTO lesions.

\section{Materials and Methods}

2.1. Study Population. From January 2017 to January 2020, 271 consecutive patients with CTO who underwent retrograde interventional therapy successfully at the People's Hospital of Liaoning Province were enrolled in this study. The patients either had failed antegrade interventional therapy or had good retrograde collateral circulation with high chances of success with the primary retrograde interventional therapy. They were randomly divided into the ARB and non-ARB groups. All procedures were performed by Luan Bo (the corresponding author). The patients were administered loading doses of aspirin $300 \mathrm{mg}$ and clopidogrel $300 \mathrm{mg}$ (or ticagrelor $180 \mathrm{mg}$ ) before the procedure, and thereafter, aspirin $100 \mathrm{mg}$ once daily and clopidogrel $75 \mathrm{mg}$ once daily (or ticagrelor $90 \mathrm{mg}$ twice daily) as dual antiplatelet treatment regimen. The routine access for the procedure was the radial artery, and the physician determined the access as needed. During the procedure, a standard dose of unfractionated heparin $(100 \mathrm{IU} / \mathrm{kg})$ was administered for anticoagulant therapy, and $2000 \mathrm{IU}$ was added every hour. The use of glycoprotein IIb/IIIa inhibitor (GPI) was left to the physician's discretion. The study was approved by the Institutional Review Board of the People's Hospital of Liaoning Province. This study complied with the Declaration of Helsinki, and all patients signed informed consent before participation.

2.2. Definitions. CTO was defined as thrombolysis in myocardial infarction (TIMI) grade-0 flow for a duration of more than 3 months, angiographically documented or clinically defined [4]. CC grade was defined by Werner as follows: $\mathrm{CC} 0$, no continuous connection between donor and recipient vessel; $\mathrm{CC} 1$, continuous thread-like connection; and CC2, continuous, small side branch-like connection [5]. $\mathrm{CC}$ tortuosity was defined as the presence of more than two successive curves (within $2 \mathrm{~mm}$ ) bent by $>180^{\circ}$ within a segment and less than three times the diameter of the collateral channel [6]. Adverse channel entry and exit angles were defined as a cut-off angle $<90^{\circ}$ [6]. For evaluating the antegrade PCI difficulty, the J-CTO score was used [7].
Retrograde guidewire tracking success was defined as the guidewire reaching the distal end of the occluded segment through collateral circulation without causing serious complications. Procedural success was defined as residual stenosis $<30 \%$ and TIMI grade 3 for distal blood flow after stenting, without serious complications. Severe complications were defined as coronary artery perforation, dissection, hematoma, cardiac arrest, and pericardial tamponade, among others, requiring pericardiocentesis. Retrograde guidewire tracking time was defined as the time in which the guidewire entered from the retrograde CC ostial to the distal end of the occluded segment. Retrograde microcatheter tracking time was defined as the time from entry of the microcatheter at the CC ostial until it reaches the distal end of the occluded segment. Retrograde time was defined as the time from entry of the retrograde guidewire at the CC ostial until it reaches the antegrade guidewire, while passing through the occluded segment. All imaging data were recorded in detail and evaluated by two senior cardiologists. When their opinions differed, a third expert's opinion was sought.

2.3. Interventional Procedures. Procedural access was determined by the operator. Bilateral radiography was carried out for all patients to comprehensively evaluate the CTO lesions. The selection of collateral circulation, as well as the use and replacement of guidewires, was decided by the operator. First, a floppy wire carrying a $150 \mathrm{~cm}$ microcatheter was used to enter the $\mathrm{CC}$ ostial. When the guidewire reached the distal end of the occluded segment through the $\mathrm{CC}$, the microcatheter was advanced to the distal end of the occluded segment, and then, the selection of the retrograde guidewire was at the operator's decision. For short lesions, retrograde wire crossing could be attempted. For long lesions, reverse controlled antegrade or retrograde subintimal tracking (CART) could be adopted.

2.4. Statistical Analysis. Statistical Package for the Social Sciences (SPSS) for Windows (version 20; IBM SPSS Inc., Chicago, IL, USA) was used for the statistical analysis. All normally distributed continuous data are expressed as mean $\pm S D$, and those not normally distributed are expressed as median (range). Categorical variables are presented as numbers and percentages. To compare continuous variables, Student's $t$-test or Mann-Whitney $U$ test was used. To compare categorical variables, the chi-square test and Fisher exact test were used in the case of sparse data. All tests were two-sided, and $p$ values of $<0.05$ were considered significant.

\section{Results}

3.1. Baseline Characteristics. A total of 271 patients with CTO who underwent retrograde interventional therapy were included in this study. There was no significant difference in age, sex, body mass index, diabetes mellitus, hypertension, dyslipidemia, current smoker status, previous myocardial infarction, previous $\mathrm{PCI}$, previous CABG, and LVEF $(p>0.05)$ (Table 1). 
TABLE 1: Clinical characteristics of study population.

\begin{tabular}{lccc}
\hline Variables & ARB group $(n=129)$ & Non-ARB group $(n=142)$ & $p$ value \\
\hline Age (years) & $65.8 \pm 8.7$ & $66.2 \pm 9.1$ & 0.427 \\
Gender (female), $n(\%)$ & $52(40.3 \%)$ & $55(38.7 \%)$ & 0.805 \\
Body mass index $\left(\mathrm{kg} / \mathrm{m}^{2}\right)$ & $22.7 \pm 2.9$ & $23.1 \pm 3.5$ & 0.394 \\
Diabetes mellitus, $n(\%)$ & $42(32.6 \%)$ & $45(31.7 \%)$ & 0.897 \\
Hypertension, $n(\%)$ & $39(30.2 \%)$ & $48(33.8 \%)$ & 0.603 \\
Dyslipidaemia on admission, $n(\%)$ & $38(29.5 \%)$ & $66(46.2 \%)$ & $0.8 \%)$ \\
Current smoker, $n(\%)$ & $61(47.3 \%)$ & $40(28.2 \%)$ & 0.904 \\
Previous MI, $n(\%)$ & $35(27.1 \%)$ & $101(71.1 \%)$ & 0.892 \\
Previous PCI, $n(\%)$ & $96(74.4 \%)$ & $12(8.5 \%)$ & 0.586 \\
Previous CABG, $n(\%)$ & $9(7.0 \%)$ & $41.7 \pm 7.5$ & 0.821 \\
LVEF $(\%)$ & $42.1 \pm 7.2$ & 0.717 \\
\hline
\end{tabular}

MI, myocardial infarction; PCI, percutaneous coronary intervention; CABG, coronary artery bypass grafting; LVEF, left ventricular ejection fraction.

3.2. Angiographic and Procedural Characteristics. The femoral/radial approach was the most used access, accounting for $68.6 \%(186 / 271)$ of total cases. The right coronary artery was the most commonly involved in CTO lesions, accounting for $64.2 \%(171 / 271)$ of total cases. Angiography results showed that there was no significant difference in the procedural access, CTO vessels, in-stent CTO ratio, lesion characteristics (blunt stump at proximal cap, calcification, occlusion length $>20 \mathrm{~mm}$, side branch at landing zone, and J-CTO score), and CC characteristics (CC tortuosity and Werner score 0-1) $(p>0.05)$ (Table 2). Primary retrograde approach accounted for $73.8 \%(200 / 271)$ of cases, $69.0 \%$ (187/271) using the septal branch, and 31.0\% (84/271) epicardial collateral branch. There was no significant difference between the two groups in terms of the adoption of the septal branch, epicardial collateral branch, or primary retrograde approach $(p>0.05)$ (Table 3$)$. Although the time of wire CC tracking in the ARB group was shorter than in the non-ARB group ( $25.4 \pm 8.5$ vs. $26.4 \pm 9.7, p=0.348$ ), there was no significant difference. The time of the retrograde microcatheter tracking $(10.2 \pm 3.8$ vs. $15.5 \pm 6.8, p=0.012)$ and time of the retrograde approach $(62.8 \pm 20.3$ vs. $70.4 \pm 24.3$, $p=0.026)$ in the ARB group were significantly shorter than those of the non-ARB group. The radiation dose (223.6 \pm 112.7 vs. $295.2 \pm 129.3, p=0.028)$ and fluoroscopy time $(50.6 \pm 21.3$ vs. $62.3 \pm 32.1, p=0.030)$ in the ARB group were significantly lower than those of the non-ARB group. The contrast volume $(301.8 \pm 146.7$ vs. $352.2 \pm 179.5$, $p=0.032)$ in the ARB group was significantly lower than that in the non-ARB group. There was no significant difference between the two groups for the antegrade Guidezilla usage or reverse CART $(p>0.05)$ (Table 3).

There was no significant difference in procedural complications or in-hospital major adverse cardiac and cerebral events (MACCE). In the ARB group, no donorvessel dissection occurred. In the non-ARB group, the incidence of donor-vessel dissection was 1.4\% (2/142), and all cases were coronary artery ostial injury caused by guiding; these patients underwent stent implantation. No patient in the ARB group experienced CC perforation, while the incidence of CC perforation in the non-ARB group was $0.7 \%$ (1/142). The incidence of target-vessel perforation in the ARB group was $0.8 \%(1 / 129)$, while that of the non-ARB group was $0.7 \%(1 / 142)$. No cardiac tamponade was documented, and no death occurred in either group. The incidence of acute stent thrombosis in the ARB group was $0.8 \%$ (1/129). Target-vessel revascularization (TVR) was $0.8 \%(1 / 129)$ in the ARB group and $0.7(1 / 142)$ in the nonARB group (Table 4).

\section{Discussion}

This is the first study to confirm that the ARB technique can significantly improve the efficiency of retrograde PCI for patients with CTO. Its advantages include a shorter procedural time and a lower X-ray and contrast agent exposure without increasing procedure-related complications.

Septal branch or epicardial collateral circulation could be used in retrograde CTO-PCI. Several studies have confirmed that septal collateral circulation is safer than epicardial collateral circulation although the proportion of retrograde interventional therapy via epicardial collateral circulation has gradually increased in recent years. However, studies have confirmed that retrograde interventional therapy via epicardial collateral circulation has the same effectiveness and safety [8]. Guidewire tracking in a CC is a key step during retrograde CTO-PCI [7]. Previous studies have confirmed that vessel size, severe collateral tortuosity, side branch at CC tortuosity, inadequate CC exit, and Werner CC score were predictors of retrograde wire CC tracking failure [8-10]. Some studies have confirmed that the incidence of initial microcatheter CC tracking success was $77.5 \%$ in 280 procedures and that retrograde guiding support is an important factor for the success of microcatheter tracking [11].

In CTO-PCI, the guiding catheter must provide strong support for the passing of guidewire or for the subsequent advancement of microcatheter, followed by the balloon and stent. Therefore, the selection of a guiding catheter with a large lumen, strong support, and good coaxiality is a prerequisite for a successful CTO-PCI. In general, EBU, XB, and AL series of guiding catheters are used for CTO lesions in the left anterior descending branch, while AL series of guiding catheters are used for CTO lesions in the left circumflex coronary artery. For all CTO lesions in the right coronary artery, AL series of guiding catheters are considered. In 
TABle 2: Angiographic characteristics of the study population.

\begin{tabular}{|c|c|c|c|}
\hline Variables & ARB group $(n=129)$ & Non-ARB group $(n=142)$ & $p$ value \\
\hline \multicolumn{4}{|l|}{ Access site } \\
\hline Radial only & $13(10.1 \%)$ & $18(12.7 \%)$ & 0.569 \\
\hline Femoral + radial & $90(69.8 \%)$ & $96(67.6 \%)$ & 0.793 \\
\hline Femoral only & $26(20.2 \%)$ & $30(21.1 \%)$ & 0.881 \\
\hline \multicolumn{4}{|l|}{ CTO vessel } \\
\hline LAD-CTO, $n(\%)$ & $37(28.7 \%)$ & $38(26.8 \%)$ & 0.786 \\
\hline LCX-CTO, $n(\%)$ & $10(7.8 \%)$ & $14(9.9 \%)$ & 0.671 \\
\hline RCA-CTO, $n(\%)$ & $82(63.6 \%)$ & $92(64.8 \%)$ & 0.899 \\
\hline \multicolumn{4}{|l|}{ Lesion characteristics } \\
\hline In-stent CTO, $n(\%)$ & $13(9.4 \%)$ & $16(11.3 \%)$ & 0.696 \\
\hline Blunt stump at proximal cap & $58(45.0 \%)$ & $65(45.3 \%)$ & 0.903 \\
\hline Calcification & $52(40.3 \%)$ & $55(38.7 \%)$ & 0.805 \\
\hline CC tortuosity, $n(\%)$ & $48(37.2 \%)$ & $52(36.6 \%)$ & 1 \\
\hline Occlusion length $>20 \mathrm{~mm}$ & $102(79.1 \%)$ & $103(72.5 \%)$ & 0.257 \\
\hline Side branch at landing zone & $58(45.0 \%)$ & $60(42.3 \%)$ & 0.713 \\
\hline J-CTO score & $3.0 \pm 1.1$ & $3.1 \pm 1.2$ & 0.428 \\
\hline Werner score 0 & $9(7.0 \%)$ & $10(7.0 \%)$ & 1 \\
\hline Werner score 1 & $57(44.2 \%)$ & $70(49.3 \%)$ & 0.465 \\
\hline Werner score 2 & $63(48.8 \%)$ & $62(43.7 \%)$ & 0.464 \\
\hline
\end{tabular}

CTO, chronic total occlusion; LAD, left anterior descending artery; LCX, left circumflex artery; RCA, right coronary artery; CC, collateral channel.

TABle 3: Procedural characteristics of the study population.

\begin{tabular}{|c|c|c|c|}
\hline Variables & ARB group $(n=129)$ & Non-ARB group $(n=142)$ & $p$ value \\
\hline Septal CC, $n(\%)$ & $92(71.3 \%)$ & $95(66.9 \%)$ & 0.511 \\
\hline Epicardial CC, $n(\%)$ & $37(28.7 \%)$ & $47(33.1 \%)$ & 0.511 \\
\hline Primary retrograde approach, $n(\%)$ & $99(76.7 \%)$ & $101(71.1 \%)$ & 0.334 \\
\hline After failed antegrade approach, $n(\%)$ & $30(23.3 \%)$ & $41(28.9 \%)$ & 0.334 \\
\hline Time of wire CC tracking (min) & $25.4 \pm 8.5$ & $26.4 \pm 9.7$ & 0.348 \\
\hline Time of retrograde microcatheter tracking ( $\mathrm{min})$ & $10.2 \pm 3.8$ & $15.5 \pm 6.8$ & 0.012 \\
\hline Time of retrograde approach $(\mathrm{min})$ & $62.8 \pm 20.3$ & $70.4 \pm 24.3$ & 0.026 \\
\hline Radiation dose (Gy. $\left.\mathrm{cm}^{2}\right)$ & $223.6 \pm 112.7$ & $295.2 \pm 129.3$ & 0.028 \\
\hline Fluoroscopy time (min) & $50.6 \pm 21.3$ & $62.3 \pm 32.1$ & 0.030 \\
\hline Antegrade Guidezilla usage, $n(\%)$ & $40(31.0 \%)$ & $47(33.1 \%)$ & 0.795 \\
\hline Retrograde wire crossing, $n(\%)$ & $14(10.9 \%)$ & $13(9.2 \%)$ & 0.688 \\
\hline Reverse CART, $n(\%)$ & $115(89.1 \%)$ & $129(90.8 \%)$ & 0.688 \\
\hline Contrast volume $(\mathrm{ml})$ & $301.8 \pm 146.7$ & $352.2 \pm 179.5$ & 0.032 \\
\hline
\end{tabular}

CC, collateral channel; CART, controlled antegrade and retrograde tracking. Bold values are statistically significant $(p<0.05)$.

TABLE 4: In-hospital outcomes.

\begin{tabular}{|c|c|c|c|}
\hline Variables & ARB group $(n=129)$ & Non-ARB group $(n=142)$ & $p$ value \\
\hline \multicolumn{4}{|l|}{ Procedural complications } \\
\hline Donor-vessel dissection, $n(\%)$ & $0(0 \%)$ & $2(1.4 \%)$ & 0.499 \\
\hline CC perforation & $0(0 \%)$ & $1(0.7 \%)$ & 1 \\
\hline Target-vessel perforation, $n(\%)$ & $1(0.8 \%)$ & $1(0.7 \%)$ & 1 \\
\hline Cardiac tamponade, $n(\%)$ & $0(0 \%)$ & $0(0 \%)$ & - \\
\hline \multicolumn{4}{|l|}{ In-hospital MACCE } \\
\hline All-cause mortality, $n(\%)$ & $0(0 \%)$ & $0(0 \%)$ & - \\
\hline Acute in-stent thrombosis, $n(\%)$ & $1(0.8 \%)$ & $0(0 \%)$ & 0.476 \\
\hline TVR, $n(\%)$ & $1(0.8 \%)$ & $1(0.7 \%)$ & 1 \\
\hline
\end{tabular}

CC, collateral channel; MACCE, major adverse cardiac and cerebral event; TVR, target-vessel revascularization.

contrast, JL and JR series of guiding catheters are rarely used for CTO lesions. However, rarely does an appropriate guiding catheter combine strong support and good coaxiality together. There are also other equipment and techniques to increase catheter support, such as the motherand-child catheter, OTW catheter, deep catheter insertion, microcatheter, balloon anchoring, double guidewires, and multiple guidewires. However, problems can arise, such as 
intertwining of double and multiple guidewires, damage of branch vessels, perforation and dissection of the coronary arteries during balloon anchoring, and coronary artery ostial damage due to deep catheter insertion.

Guidezilla extension catheter provides stronger guiding support to facilitate the delivery of devices. It also increases coaxiality in order to (a) prevent the catheter from slipping accidentally, (b) overcome the different axes caused by arterial vascular abnormalities, and (c) prevent the catheter from exiting during the procedure.

At the same time, Guidezilla extension catheter prevents coronary artery ostial damage caused by strong guiding catheters and allows the use of guiding catheters with strong support and good coaxiality in CTO-PCI. However, guiding catheters with both qualities will increase the probability of coronary ostial damage, and deep insertion of guiding catheters carries a higher risk of coronary ostial and sinus damage. Guidezilla extension catheter together with an ordinary guiding catheter may not only prevent coronary artery ostial damage caused by a strong guiding catheter, particularly in patients with coronary artery ostial lesions, but could also improve the support required for CTO-PCI, reduce procedural complications, and increase the safety of the procedure. In this study, two cases of donor-vessel dissection occurred in the non-ARB group, both of which were due to coronary artery ostial dissection arising from the deep insertion of the catheter and were treated by stent implantation. The proportion of antegrade use of Guidezilla was similar between the two groups of patients. CTO lesions are often accompanied by tortuous, calcified, and diffuse lesions, as well as distal lesions [12], which increase the difficulty in guidewire tracking, as well as subsequent balloon and stent passing. The use of Guidezilla extension catheter enhances guiding support, improves coaxiality, and facilitates the entry of devices. In contrast, the use of antegrade Guidezilla is convenient for the retrograde guidewire to enter the antegrade guiding catheter, which is known as the active greeting technique (AGT) [13]. This study confirmed that the ARB technique can significantly reduce the procedural time and reduce X-ray exposure and contrast agent dosage, without increasing procedure-related complications. Combining the ARB technique with AGT can significantly improve the efficiency of retrograde interventional therapy for CTO lesions and is, thus, worthy of further promotion.

A previous meta-analysis showed that retrograde PCI for CTO lesions had a $6.9 \%$ incidence of target-vessel perforation and a $1.4 \%$ incidence of pericardial tamponade [14]. However, with advances in technology and devices, complications have steadily decreased in recent years, and the success rate has also increased significantly. This study included CTO patients who underwent successful retrograde intervention, with $0.74 \%$ (2/ 271) donor-vessel dissection and $0.74 \%(2 / 271)$ target-vessel perforation and without an occurrence of pericardial tamponade. Most procedures were performed by reverse CART mainly because the patients had higher J-CTO scores, and some of them had failed with previous antegrade interventions.

Regarding the timing of retrograde Guidezilla, we suggest the active use of retrograde Guidezilla, or ARB technique, to further improve the procedural efficiency. In order to obtain better supporting force and coaxiality, the head of
Guidezilla entering the coronary artery should be sufficiently long although this may increase the risk of coronary artery injury. In particular, for patients with unstable plaques or completing balloon dilatation, we recommend that the head of Guidezilla entering the coronary artery should not exceed $15 \mathrm{~cm}$. Before advancing Guidezilla, balloon-anchoring technology is recommended, and it is important to apply small-pressure expansion to avoid coronary artery injury. PCI is recommended when the donor vessel has severe stenosis or unstable lesions before interventional treatment of CTO lesions.

\section{Limitations}

The patients included in this study were from a single center and treated by the same operator; moreover, the patients were highly selected and the sample size was relatively small, and inevitably, there are some biases. The study patients were recruited from January 2017 to January 2020; the success rate of the procedure has been increasing annually with an increase in the number of procedure and progress in instruments, which resulted in different success rates between patients treated early and those treated recently, and this could have affected the study's results. The J-CTO scores of the subjects were relatively high and were not applicable to all study populations. In the future, data from multicenter, large sample size studies conducted by highly experienced centers and operators are needed to verify our conclusions.

\section{Conclusions}

ARB is feasible, safe, and conducive to guidewire and microcatheter CC tracking in the recanalization of coronary CTO. It improves procedural efficiency and is worthy of further promotion.

\section{Data Availability}

The data supporting the results in the current study are available from the corresponding author on reasonable request.

\section{Conflicts of Interest}

The authors declare that they have no conflicts of interest.

\section{Acknowledgments}

The authors would like to thank Editage (http://www. editage.cn) for English language editing.

\section{References}

[1] P. Fefer, M. L. Knudtson, A. N. Cheema et al., "Current perspectives on coronary chronic total occlusions," Journal of the American College of Cardiology, vol. 59, no. 11, pp. 991997, 2012.

[2] T. Råmunddal, L. P. Hoebers, J. P. S. Henriques et al., "Prognostic impact of chronic total occlusions," JACC: Cardiovascular Interventions, vol. 9, no. 15, pp. 1535-1544, 2016.

[3] T. Noguchi, S. Miyazaki, I. Morii, S. Daikoku, Y. Goto, and H. Nonogi, "Percutaneous transluminal coronary angioplasty 
of chronic total occlusions, determinants of primary success and long-term clinical outcome," Catheterization and Cardiovascular Interventions, vol. 49, no. 3, pp. 258-264, 2000.

[4] G. W. Stone, D. E. Kandzari, R. Mehran et al., "Percutaneous recanalization of chronically occluded coronary arteries," Circulation, vol. 112, no. 15, pp. 2364-2372, 2005.

[5] G. S. Werner, M. Ferrari, S. Heinke et al., "Angiographic assessment of collateral connections in comparison with invasively determined collateral function in chronic coronary occlusions," Circulation, vol. 107, no. 15, pp. 1972-1977, 2003.

[6] M. B. McEntegart, A. A. Badar, F. A. Ahmad et al., "The collateral circulation of coronary chronic total occlusions," Eurointervention, vol. 11, no. 14, pp. e1596-e1603, 2016.

[7] Y. Morino, M. Abe, T. Morimoto et al., "Predicting successful guidewire crossing through chronic total occlusion of native coronary lesions within 30 minutes," JACC: Cardiovascular Interventions, vol. 4, no. 2, pp. 213-221, 2011.

[8] Z. Huang, B. Ma, B. Zhang et al., "Epicardial collateral channel for retrograded recanalization of chronic total occlusion percutaneous coronary intervention: predictors of failure and procedural outcome," Journal of Interventional Cardiology, vol. 31, no. 1, pp. 23-30, 2018.

[9] C. C. Wu, C. K. Lee, S. W. Meng et al., "Collateral channel size and tortuosity predict retrograde percutaneous coronary intervention success for chronic total occlusion," Catheteration Cardiovascular Intervention, vol. 11, no. 1, Article ID e005124, 2018.

[10] S. Rathore, O. Katoh, H. Matsuo et al., "Retrograde percutaneous recanalization of chronic total occlusion of the coronary arteries," Circulation: Cardiovascular Interventions, vol. 2, no. 2, pp. 124-132, 2009.

[11] X. Zhong, L. Ge, J. Ma et al., "Microcatheter collateral channel tracking failure in retrograde percutaneous coronary intervention for chronic total occlusion: incidence, predictors, and management," EuroIntervention, vol. 15, no. 3, pp. e253-e260, 2019.

[12] J. Karacsonyi, D. Karmpaliotis, A. Khaldoon et al., "Impact of calcium on chronic total occlusion percutaneous coronary interventions," The American Journal of Cardiology, vol. 120, no. 1, pp. 40-46, 2017.

[13] J. Ge, L. Ge, B. Zhang et al., "Active greeting technique: a mother-and-child catheter based technique to facilitate retrograde wire externalization in recanalization of coronary chronic total occlusion," Science Bulletin, vol. 63, no. 23, pp. 1565-1569, 2018.

[14] A. E. Sabbagh, V. G. Patel, O. M. Jeroudi et al., "Angiographic success and procedural complications in patients undergoing retrograde percutaneous coronary chronic total occlusion interventions: a weighted meta-analysis of 3482 patients from 26 studies," International Journal of Cardiology, vol. 174, no. 2, pp. 243-248, 2014. 